\title{
The Arabidopsis thaliana proteome harbors undiscovered multi-domain molecules with functional guanylyl cyclase catalytic centers
}

Aloysius Wong and Chris Gehring*

\begin{abstract}
Background: Second messengers link external cues to complex physiological responses. One such messenger, 3',5'-cyclic guanosine monophosphate (cGMP), has been shown to play a key role in many physiological responses in plants. However, in higher plants, guanylyl cyclases (GCs), enzymes that generate cGMP from guanosine-5'-triphosphate (GTP) have remained elusive until recently. GC search motifs constructed from the alignment of known GCs catalytic centers form vertebrates and lower eukaryotes have led to the identification of a number of plant GCs that have been characterized in vitro and in vivo.

Presentation of the hypothesis

Recently characterized GCs in Arabidopsis thaliana contributed to the development of search parameters that can identify novel candidate GCs in plants. We hypothesize that there are still a substantial number $(>40)$ of multi-domain molecules with potentially functional GC catalytic centers in plants that remain to be discovered and characterized.

Testing the hypothesis: The hypothesis can be tested, firstly, by computational methods constructing 3D models of selected GC candidates using available crystal structures as templates. Homology modeling must include substrate docking that can provide support for the structural feasibility of the GC catalytic centers in those candidates. Secondly, recombinant peptides containing the GC domain need to be tested in in vitro GC assays such as the enzyme-linked immune-sorbent assay (ELISA) and/or in mass spectrometry based CGMP assays. In addition, quantification of in vivo cGMP transients with fluorescent cGMP-reporter assays in wild-type or selected mutants will help to elucidate the biological role of novel GCS.

Implications of the hypothesis

If it turns out that plants do harbor a large number of functional GC domains as part of multi-domain enzymes, then major new insights will be gained into the complex signal transduction pathways that link cGMP to fundamental processes such as ion transport and homeostasis, biotic and abiotic stress responses as well as cGMP-dependent responses to hormones.
\end{abstract}

Keywords: 3',5'-cyclic guanosine monophosphate (cGMP), Guanosine-5'-triphosphate (GTP), Guanylyl cyclase (GC), Catalytic center, GC search motif, Homology modeling, Molecular docking

\footnotetext{
* Correspondence: christoph.gehring@KAUST.edu.sa

Division of Biological and Environmental Science and Engineering, 4700 King Abdullah University of Science and Technology, Thuwal 23955-6900, Kingdom of Saudi Arabia
}

\section{Biomed Central}

(c) 2013 Wong and Gehring; licensee BioMed Central Ltd. This is an Open Access article distributed under the terms of the Creative Commons Attribution License (http://creativecommons.org/licenses/by/2.0), which permits unrestricted use, distribution, and reproduction in any medium, provided the original work is properly cited. 


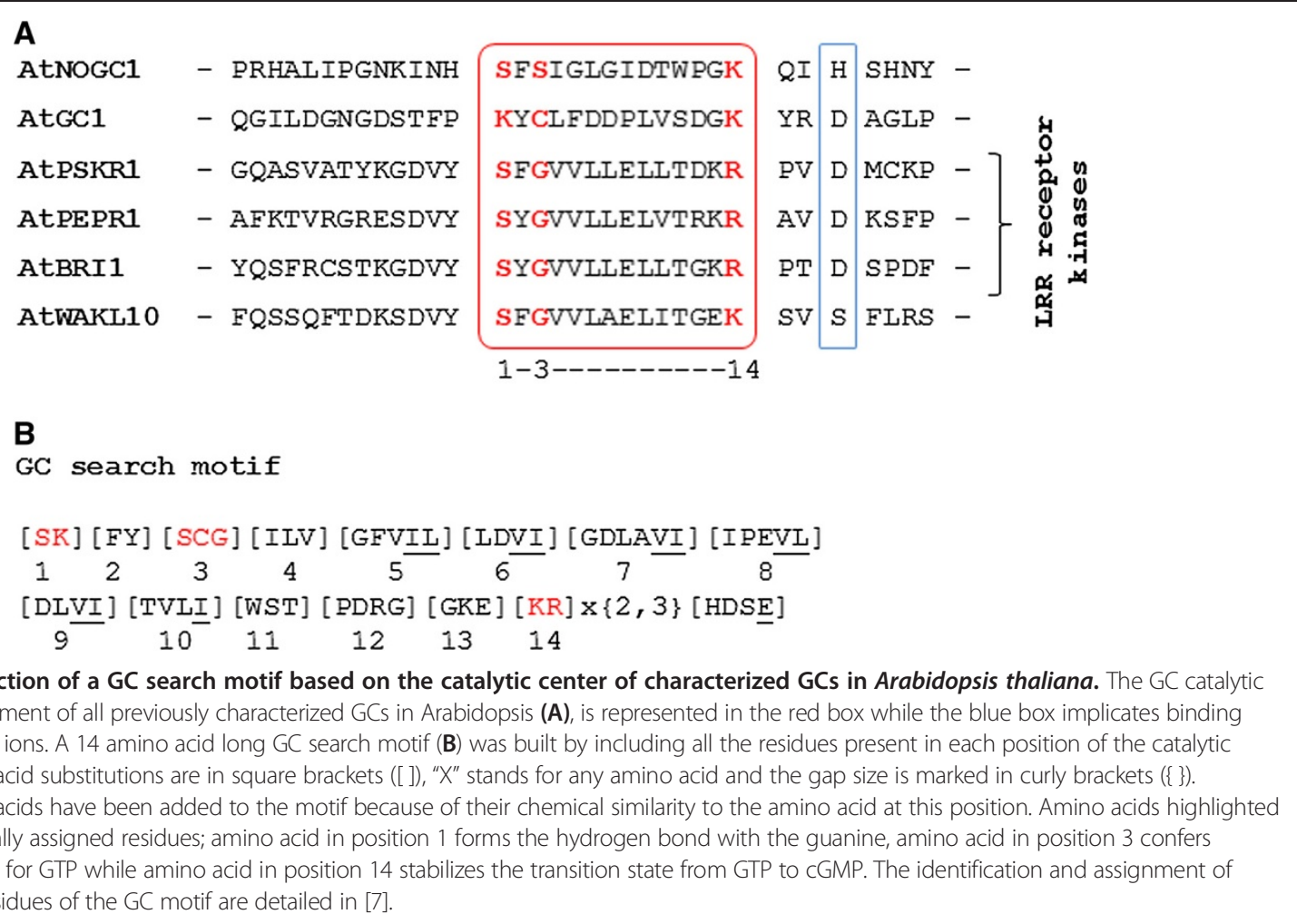

\section{Background}

While cGMP is increasingly accepted as an important signaling component in many plant responses e.g. [1-3], it is perhaps astonishing that the discovery and functional characterization of GCs in higher plants is only just beginning, particularly so since in single celled green alga Chlamydomonas reinhardtii there are $>90$ annotated nucleotide cyclases (NCs) that come in $>20$ different domain combinations with 13 different domain partners [4]. The structural diversity and complexity of
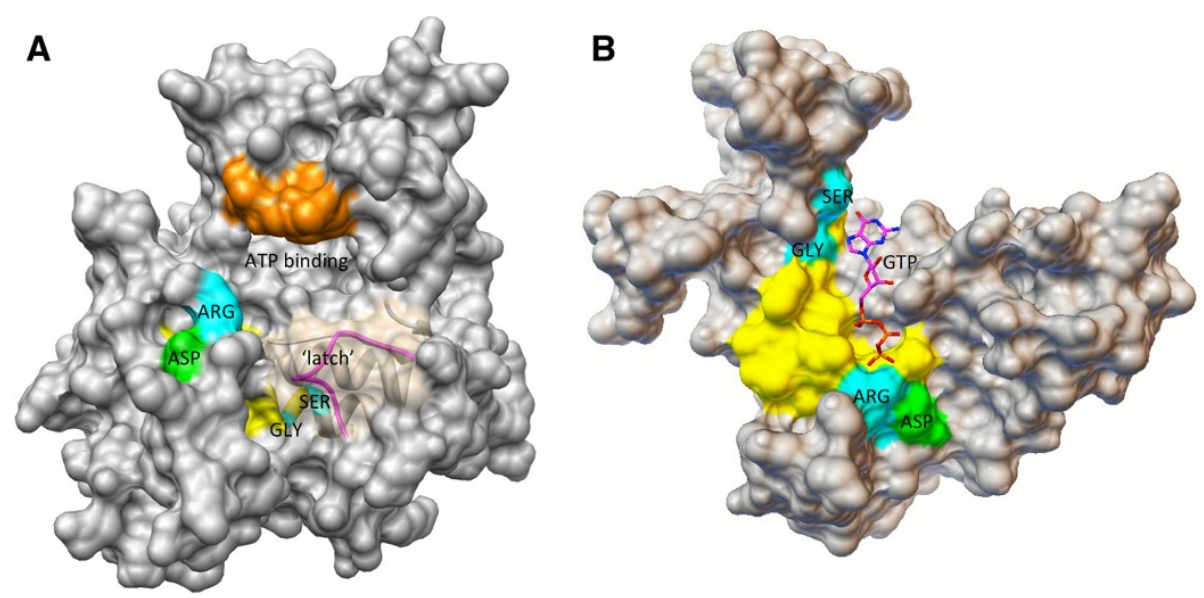

Figure 2 Computational assessment of the predicted activation states of AtPSKR1 kinase and GC catalytic centers. AtPSKR1-kinase domain (Phe ${ }^{734}-$ Val $^{1008}$ ) was modeled against the AvrPtoB-BAK1 complex (PDB entry: 3TL8) (A) and against the bacteria GC Cya2 (PDB entry: 2W01) (B) using the Modeller (ver. 9.10) software [14], and GTP docking experiments performed using AutoDock Vina (ver. 1.1.2) [15]. When AtPSKR1 assumes the kinase activation state (A), the GC catalytic center is partially covered by a nine amino acid long 'latch' that may be loosened by a molecular switch (e.g. $\mathrm{Ca}^{2+}$ ) or by forming homo- or hetero-dimers. When AtPSKR1 assumes a GC configuration (B), the GC catalytic center is completely exposed and assessable to GTP. In addition, automated docking experiments suggest feasible GTP docking. The GC catalytic center is in yellow, functionally assigned residues in the GC motif are in cyan, amino acids implicated in metal binding are in green, the ATP-binding site is in orange and the nine amino acid long 'latch' partially covering the GC catalytic center is in magenta. 


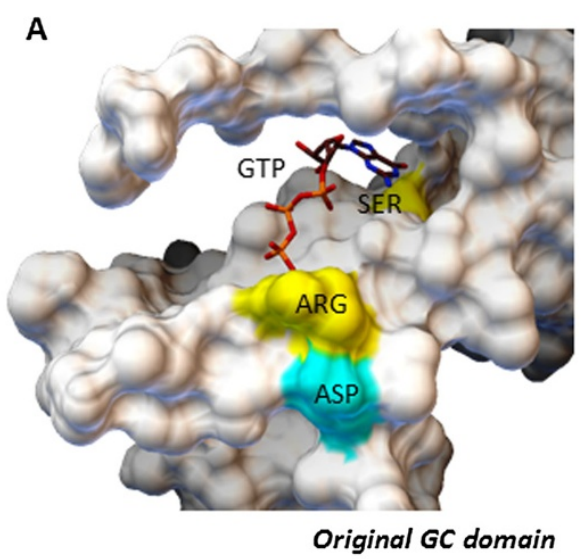

AtPSKR1 GC domain

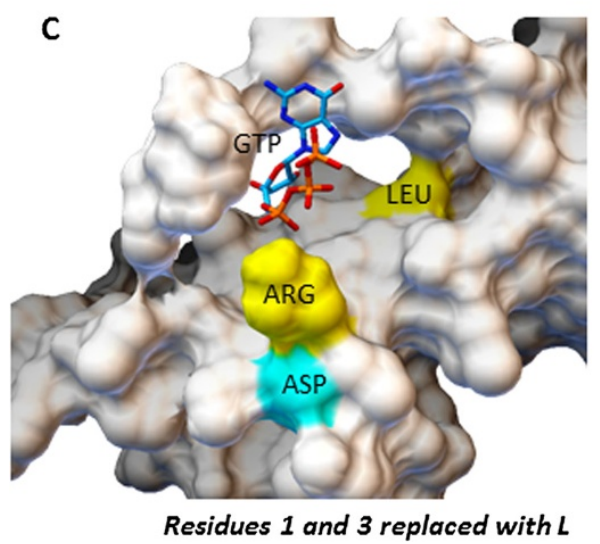

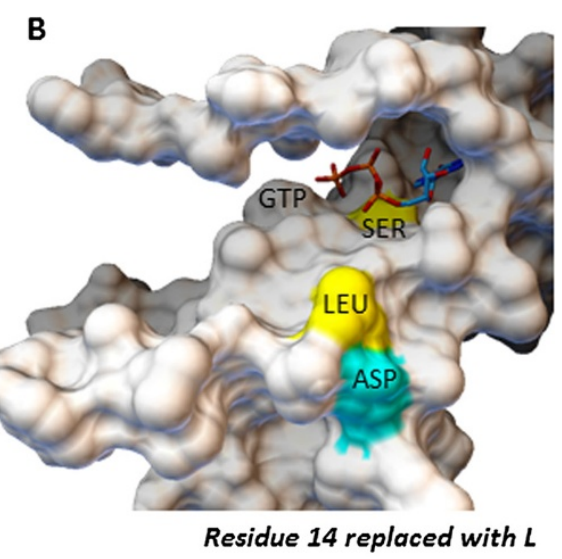
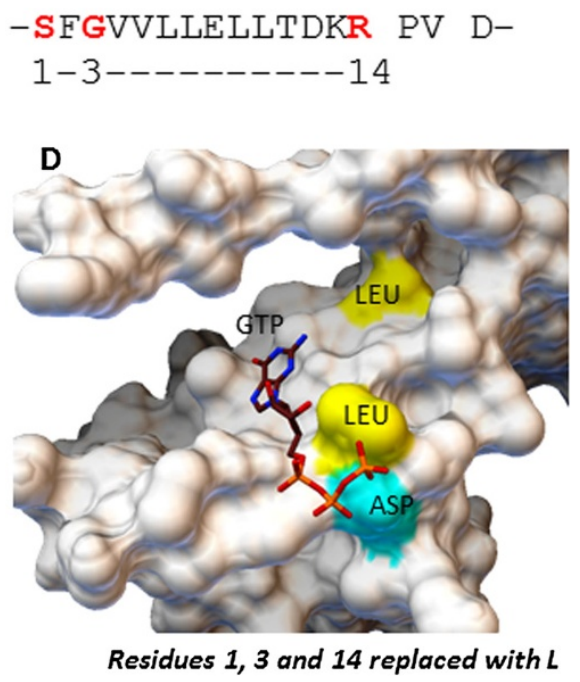

Figure 3 Assessment of the AtPSKR1 GC catalytic center by molecular docking of GTP. According to the 3D model of AtPSKR1, the GC domain is embedded within a cavity with the key residues for GTP interaction and the metal binding residue highlighted in yellow and cyan respectively. The GTP substrate docks successfully with the guanine portion at the inner-most of the cavity for interaction with the serine residue (position 1) and the phosphate end pointing outwards of the cavity towards the arginine residue (position 14) - an orientation deemed suitable for GC activity (A). When one or more key amino acid residues at position 1, 3 and/or 14 was replaced with leucine, docking experiments indicate disruptions in the docking orientations of GTP in all except for the leucine replacements at positions 1 and 3 of the GC domain. This suggests an aberrant GC catalytic activity when these key residues are replaced. GTP docking results for AtPSKR1 GC domain with leucine replacements at position 14 (B), positions 1 and 3 (C), and positions 1, 3 and 14 (D) are as illustrated. AtPSKR1-GCD (Asn $\left.{ }^{871}-G^{9}{ }^{980}\right)$ was modeled against the AvrPtoB-BAK1 complex (PDB entry: 3TL8) using the Modeller (ver. 9.10) software [14] while GTP docking experiments were performed using AutoDock Vina (ver. 1.1.2) [15]. The full-length AtPSKR1 protein and the domains organization are as shown in Additional file 2.

molecules with $\mathrm{NC}$ activity [4-6] are one likely reason why BLAST searches with known NCs from lower and higher eukaryotes did not yield candidate molecules in higher plants.

Search strategies based on conserved and functionally assigned amino acid (AA) residues in the catalytic center of known NCs [7] have now opened the way to a systematic search of NCs in higher plants and has led to the discovery of a number of Arabidopsis thaliana candidate molecules with catalytic activity in vitro and in vivo. These molecules include a wall-associated kinase like protein (AtWAKL10) with a role in defense [8], the brassinosteroid receptor (AtBRI1) [9], the Pep1 receptor (AtPepR1) [10] and the phytosulfokine receptor (AtPSKR) [11] as well as a nitric oxide-binding GC (AtNOGC1) [12]. PSKR belongs to a family of NCs that contains the GC catalytic center embedded within the intracellular kinase domain of leucine rich repeat receptor-like molecules and in in vitro experiments we have demonstrated that both the kinase and the GC domain have catalytic activity. Importantly, the natural ligands for both the PSKR and BRI1 receptors increase intracellular cGMP levels in isolated mesophyll protoplast assays suggesting that the GC activity is functionally relevant in planta $[6,11]$. 
Table 1 Molecular docking of GTP with the GC catalytic center

\begin{tabular}{lccccccc}
\hline & \multicolumn{7}{c}{ Amino acid position in the GC motif replaced with } \\
& \multicolumn{1}{c}{ leucine (L) } \\
& $\mathbf{1}$ & $\mathbf{3}$ & $\mathbf{1 4}$ & $\mathbf{1 , 3}$ & $\mathbf{1 , 1 4}$ & $\mathbf{3 , 1 4}$ & $\mathbf{1 , 3 , 1 4}$ \\
\hline AtPSKR1 & $\checkmark$ & $\checkmark$ & $\boldsymbol{x}$ & $\boldsymbol{x}$ & $\boldsymbol{x}$ & $\boldsymbol{x}$ & $\boldsymbol{x}$ \\
AtPEPR1 & $\boldsymbol{x}$ & $\boldsymbol{x}$ & $\boldsymbol{x}$ & $\boldsymbol{x}$ & $\boldsymbol{x}$ & $\boldsymbol{x}$ & $\boldsymbol{x}$ \\
AtBRI1 & $\checkmark$ & $\boldsymbol{x}$ & $\boldsymbol{x}$ & $\boldsymbol{x}$ & $\checkmark$ & $\boldsymbol{x}$ & $\boldsymbol{x}$ \\
AtWAKL10 & $\boldsymbol{x}$ & $\boldsymbol{x}$ & $\boldsymbol{x}$ & $\boldsymbol{x}$ & $\boldsymbol{x}$ & $\boldsymbol{x}$ & $\boldsymbol{x}$
\end{tabular}

Functionally-important amino acid residues at position 1, 3 and/or 14 of the GC motif were replaced with leucine for homology modeling and docking experiments.

$\checkmark$ indicates successful docking of GTP and in an orientation deemed suitable for catalysis.

$\boldsymbol{x}$ indicates unsuccessful docking of GTP or GTP docking in an orientation deemed unsuitable for catalysis.

\section{Presentation of the hypothesis}

We propose that in addition to the six characterized GCs in Arabidopsis thaliana to-date (AtGC1, AtNOGC1, AtPSKR1, AtPEPR1, AtBRI1 and AtWAKL10), higher plants harbor a substantially larger number of GCs that remain to be discovered. This hypothesis is based on the fact that the tested GCs share a distinct AA signature in the catalytic center (Figure 1A) where the AA at position 1 forms the hydrogen bond with the guanine, the residue in position 3 confers substrate specificity for GTP while the AA in position 14 stabilizes the transition state from GTP to cGMP and two or three AAs away from the Cterminal end of the motif is the residue that interacts with the $\mathrm{Mg}^{2+} / \mathrm{Mn}^{2+}$ ions (Figure 1B). This motif based on tested GCs (Figure 1B) identifies 41 novel Arabidopsis candidate GCs (see Additional file 1) (http://www. arabidopsis.org/cgi-bin/patmatch/nph-patmatch.pl) [13] and no proteins in kingdoms other than the Viridiplantae. However, we do not predict that all retrieved candidate GCs will have activity in vitro and/or in vivo, nor do we exclude that molecules that do not contain the motif function as GCs.

\section{Testing the hypothesis}

Firstly, the hypothesis can be tested by using computational approaches to predict the structural properties of candidate GCs and testing should include automated substrate (GTP) docking protocols. To obtain insights on the structural features of the GC catalytic centers, $3 \mathrm{D}$ models can be constructed using readily available crystal structures as templates. A high sequence similarity between subject and template will generate accurate $3 \mathrm{D}$ models. While sequence similarity of $25 \%$ is sufficient for structural predictions, we recommend selecting templates with a BLAST alignment score of at least 50-80 (color key: green). For example, a 3D structure of the GC region of AtPSKR1 built using homology modeling techniques revealed that the GC catalytic center is embedded within a cavity, presumably providing ideal steric interactions for substrate docking. While selecting template structures with the highest sequence similarity to the candidate GCs is the common practice, we would recommend to also evaluate the candidate proteins against a known GC template (e.g. crystal structure of a bacterial or human GC) since many plant candidate GCs are embedded within kinases, and modeling against a kinase template may not reflect the configuration of an activated candidate GC. The reason is that dual-activity enzymes such as AtPSKR1 do not assume concurrent activation states for both the kinase and GC catalytic domains, and it is likely that a molecular switch (e.g. $\mathrm{Ca}^{2+}$ and/or dimerization with another molecule) is required to shift from kinase to GC activation. In the example here, when the AtPSKR1 molecule is in the kinase state the GC catalytic center is partially buried. In turn, when the molecule is in the GC configuration, the GC catalytic center is completely exposed and GTP can successfully dock (as predicted by AutoDock Vina) (Figure 2). GTP docking at the PSKR1 GC center in the correct orientation favorable for interactions with the key residues within the cavity is represented in Additional file 2: Figure 3A. Functionally assigned residues of the $\mathrm{GC}$ motif can be replaced with another amino acid to estimate the importance or relevance of these residues in maintaining a functional configuration of the catalytic center. For example, when one or more key residues in the AtPSKR1 GC domain is replaced with leucine, GTP is more likely to fail to dock or dock in the wrong orientation, implying compromised or abolished GC activity (Figure 3B-D). In previously characterized Arabidopsis GCs, GTP docking is also disrupted when key residues are replaced (Table 1). We note that these computational methods alone are not diagnostic of GC activity and do not distinguish GCs from other enzymes that also catalyze GTP (e.g. GTPases). They however lend good support to the experimental data and can be used as an initial screen to assist in the selection of candidate molecules from a potentially large pool of proteins for subsequent in vitro and/or in vivo enzymatic functional assays.

Secondly, the candidate GCs need to be tested in vitro by incubating the recombinant protein harboring the GC domain with GTP and the appropriate metal ions $\left(\mathrm{Mg}^{2+}\right.$ and/or $\left.\mathrm{Mn}^{2+}\right)$. This recombinant protein can be made by molecular cloning methods with the DNA construct expressed in an E. coli host system and affinity purified for the following in vitro GC activity testing. GC enzymatic reaction is initiated by incubating the purified recombinant protein in buffer containing the 
aforementioned ingredients. cGMP generation can then be measured using commercially available cGMP immunoassay kits which will provide an indication of GC activity. Cyclic GMP production should be further verified using mass spectrometry based techniques that consistently record higher cGMP amounts in independent in vitro experiments than those obtained with ELISA-based assays [9]. The substantially lower in vitro activities of plant GCs compared to animal GCs [5] have raised concerns regarding (1) the folding and structural integrity of the recombinant plant GCs and (2) the reliability of the in vitro recombinant GC activity assays to detect such low amounts of cGMP $[16,17]$. We therefore recommend that candidate GCs be evaluated with both the biochemical assays and the more sensitive mass spectrometric methods.

Thirdly, candidate GC candidates should also be studied in vivo [18] with fluorescent cGMP-reporter assays, since this is a direct way to link ligand-binding to receptor-coupled GCs, and to the generation of cGMP and cGMP-dependent downstream effects.

\section{Implications of the hypothesis}

Currently, we know that a growing number of fundamental physiological processes, including gating of ion channels [19], specific phosphorylation events [18], post-translational modifications [20], stomatal guard cell movements and responses to hormones [18,21] all depending, at least in part, on cGMP. Consequently, a major outstanding question is, where are the enzymes that catalyze the reaction from GTP to cGMP, how many are there in e.g. Arabidopsis, and how are they regulated? If our hypothesis proves right, and novel multi-domain enzymes with stimulus- and/or ligand-specific GC activity will be discovered, we will be able to finally unravel the complex signal transduction networks that link environmental stimuli to cGMP-dependent responses in plants such as chloroplast development and anthocyanin synthesis [22,23]. Detailed analysis of cGMP-dependent responses will have to include a genetic and molecular analysis of transgenic plants with overexpressing or knocked-down of candidate GCs as well as transcriptomics studies that reveal further aspects and the extent to which cGMP modulates down-stream effects. In addition, and given the importance of cGMP-dependent phosphorylation, we would argue that comparative phospho-proteomics of wild type and GC mutants will provide a systems view of specific phosphorylation cascades that are induced by the activation of target GCs. Taken together, we predict, that if our hypothesis is true and the candidate GCs will be analyzed in considerable depth, it will establish cGMP, much like cytosolic free $\mathrm{Ca}^{2+}$, as a key second messenger in plant responses.

\section{Additional files}

Additional file 1: The file contains the pattern matching (patmatch) search parameters and a list of the 41 new candidates retrieved using the proposed GC motif.

Additional file 2: The file contains a model of the full-length AtPSKR1 with the respective LRR, kinase and GC domains represented.

\section{Abbreviations}

cGMP: 3',5'-cyclic guanosine monophosphate; GTP: Guanosine-5'-triphosphate; GC: Guanylyl cyclase; BLAST: Basic local alignment search tool; ELISA: Enzyme-linked immune-sorbent assay.

\section{Competing interests}

The authors declare that they have no competing interests.

\section{Authors' contributions}

CG conceived the project. AW performed the modeling and bioinformatics analysis, and CG and AW wrote the manuscript. All authors read and approved the final manuscript.

Received: 4 May 2013 Accepted: 5 July 2013

Published: 8 July 2013

\section{References}

1. Donaldson L, Ludidi N, Knight MR, Gehring C, Denby K: Salt and osmotic stress cause rapid increases in Arabidopsis thaliana cGMP levels. FEBS Lett 2004, 569:317-320.

2. Maathuis FJ: cGMP modulates gene transcription and cation transport in Arabidopsis roots. Plant J 2006, 45:700-711.

3. Pasqualini S, Meier S, Gehring C, Madeo L, Fornaciari M, Romano B, Ederli L: Ozone and nitric oxide induce cGMP-dependent and -independent transcription of defence genes in tobacco. New Phytol 2009, 181:860-870.

4. Meier S, Seoighe C, Kwezi L, Irving H, Gehring C: Plant nucleotide cyclases: an increasingly complex and growing family. Plant Signal Behav 2007, 2:536-539.

5. Gehring C: Adenyl cyclases and CAMP in plant signaling - past and present. Cell Commun Signal 2010, 8:15

6. Irving HR, Kwezi L, Wheeler J, Gehring C: Moonlighting kinases with guanylate cyclase activity can tune regulatory signal networks. Plant Signal Behav 2012, 7:201-204.

7. Ludidi N, Gehring C: Identification of a novel protein with guanylyl cyclase activity in Arabidopsis thaliana. J Biol Chem 2003, 278:6490-6494.

8. Meier S, Ruzvidzo O, Morse M, Donaldson L, Kwezi L, Gehring C: The Arabidopsis wall associated kinase-like 10 gene encodes a functional guanylyl cyclase and is co-expressed with pathogen defense related genes. PloS One 2010, 5:e8904.

9. Kwezi L, Meier S, Mungur L, Ruzvidzo O, Irving H, Gehring C: The Arabidopsis thaliana brassinosteroid receptor (AtBRI1) contains a domain that functions as a guanylyl cyclase in vitro. PLoS One 2007, 2:e449.

10. Qi Z, Verma R, Gehring C, Yamaguchi Y, Zhao Y, Ryan CA, Berkowitz GA: $\mathrm{Ca}^{2+}$ signaling by plant Arabidopsis thaliana Pep peptides depends on AtPepR1, a receptor with guanylyl cyclase activity, and cGMP-activated $\mathrm{Ca}^{2+}$ channels. Proc Natl Acad Sci U S A 2010, 107:21193-21198.

11. Kwezi L, Ruzvidzo O, Wheeler Jl, Govender K, lacuone S, Thompson PE, Gehring C, Irving HR: The phytosulfokine (PSK) receptor is capable of guanylate cyclase activity and enabling cyclic GMP-dependent signaling in plants. J Biol Chem 2011, 286:22580-22588.

12. Mulaudzi T, Ludidi N, Ruzvidzo O, Morse M, Hendricks N, Iwuoha E, Gehring C: Identification of a novel Arabidopsis thaliana nitric oxide-binding molecule with guanylate cyclase activity in vitro. FEBS Lett 2011, 585:2693-2697.

13. Yan T, Yoo D, Berardini TZ, Mueller LA, Weems DC, Weng S, Cherry JM, Rhee SY: PatMatch: a program for finding patterns in peptide and nucleotide sequences. Nucleic Acids Res 2005, 33:W262-W266.

14. Sali A, Blundell TL: Comparative protein modelling by satisfaction of spatial restraints. J Mol Biol 1993, 234:779-815.

15. Trott O, Olson AJ: AutoDock Vina: improving the speed and accuracy of docking with a new scoring function, efficient optimization, and multithreading. J Comput Chem 2010, 31:455-461. 
16. Ashton AR: Guanylyl cyclase activity in plants? Proc Natl Acad Sci U S A 2011, 108:E96. author reply E97-98.

17. Berkowitz GA, Gehring C, Irving HR, Kwezi L: Reply to Ashton: The putative guanylyl cyclase domain of AtPepR1 and similar plant receptors. Proc Natl Acad Sci U S A 2011, 108:E97-E98.

18. Isner JC, Nuhse T, Maathuis FJ: The cyclic nucleotide cGMP is involved in plant hormone signalling and alters phosphorylation of Arabidopsis thaliana root proteins. J Exp Bot 2012, 63:3199-3205.

19. Zelman AK, Dawe A, Gehring C, Berkowitz GA: Evolutionary and structural perspectives of plant cyclic nucleotide-gated cation channels. Front Plant Sci 2012, 3:95

20. Marondedze C, Turek I, Parrott B, Thomas L, Jankovic B, Lilley KS, Gehring C: Structural and functional characteristics of CGMP-dependent methionine oxidation in Arabidopsis thaliana proteins. Cell Commun Signal 2013, 11:1.

21. Pharmawati M, Billington T, Gehring CA: Stomatal guard cell responses to kinetin and natriuretic peptides are CGMP-dependent. Cell Mol Life Sci 1998, 54:272-276

22. Bowler C, Yamagata H, Neuhaus G, Chua NH: Phytochrome signal transduction pathways are regulated by reciprocal control mechanisms. Genes Dev 1994, 8:2188-2202.

23. Bowler C, Neuhaus G, Yamagata H, Chua NH: Cyclic GMP and calcium mediate phytochrome phototransduction. Cell 1994, 77:73-81.

doi:10.1186/1478-811X-11-48

Cite this article as: Wong and Gehring: The Arabidopsis thaliana proteome harbors undiscovered multi-domain molecules with functional guanylyl cyclase catalytic centers. Cell Communication and Signaling 2013 11:48

\section{Submit your next manuscript to BioMed Central and take full advantage of:}

- Convenient online submission

- Thorough peer review

- No space constraints or color figure charges

- Immediate publication on acceptance

- Inclusion in PubMed, CAS, Scopus and Google Scholar

- Research which is freely available for redistribution 